Case Report

\title{
Galactose-Deficient IgA1 Deposits in Clear Cell Renal Cell Carcinoma-Related Henoch-Schönlein Purpura Nephritis
}

\author{
Yuhong Zhao, ${ }^{1}$ Youngki Kim, ${ }^{2}$ Milind Junghare, ${ }^{3}$ Viral Vakil, ${ }^{3}$ Xuesong Su, ${ }^{4}$ Faqian Li, $^{5}$ \\ and Lihong Bu $\mathbb{C}^{5}$ \\ ${ }^{1}$ Department of Nephrology, Central Hospital Affiliated to Shenyang Medical College, Shenyang, Liaoning 110024, China \\ ${ }^{2}$ Department of Pediatrics, Division of Pediatric Nephrology, University of Minnesota, Minneapolis, MN 55455, USA \\ ${ }^{3}$ Department of Renal Disease and Hypertension, University of Minnesota, Minneapolis, MN 55455, USA \\ ${ }^{4}$ Department of Nephrology, Shengjing Hospital of China Medical University, Shenyang, Liaoning 110004, China \\ ${ }^{5}$ Department of Laboratory Medicine and Pathology, University of Minnesota, Minneapolis, MN 55455, USA
}

Correspondence should be addressed to Lihong Bu; lbu@umn.edu

Received 20 June 2020; Accepted 4 August 2020; Published 24 August 2020

Academic Editor: Ze'ev Korzets

Copyright (c) 2020 Yuhong Zhao et al. This is an open access article distributed under the Creative Commons Attribution License, which permits unrestricted use, distribution, and reproduction in any medium, provided the original work is properly cited.

\begin{abstract}
Recent studies suggest that galactose-deficient IgA1 (Gd-IgA1) plays a role in the pathogenesis of primary IgA nephropathy (IgAN) and Henoch-Schönlein purpura nephritis (HSPN). Furthermore, immunostaining of KM55, an antibody that identifies Gd-IgA1, may be helpful to differentiate primary IgAN and HSPN from secondary causes of glomerular IgA deposition. We report sequential kidney biopsies of a malignancy-associated HSPN, showing intense glomerular mesangial IgA deposition at the initial kidney biopsy and dramatic decrease in disappearance of glomerular deposits after tumor removal. We demonstrate that the glomerular IgA deposition contains Gd-IgA1, detected by immunostaining of KM55, with similar distribution and intensity to IgA. This suggests that renal Gd-IgA1 deposition may play a role in the pathogenesis of malignancy-associated HSPN.
\end{abstract}

\section{Introduction}

Henoch-Schönlein purpura (HSP), also referred as immunoglobulin A vasculitis (IgAV), is the most common systemic vasculitis involving the small blood vessels in pediatric population with kidney involvement present in 30-50\% [1]. Similar to IgA nephropathy (IgAN), Henoch-Schönlein purpura nephritis (HSPN) is characterized by the deposition of IgA dominant or codominant immune complex in the glomerular mesangial and capillary wall regions. The glomerular IgA deposition in IgAN is predominantly of the IgA1 subclass [2]. Galactose-deficient IgA1 (Gd-IgA1) has been identified to be a key element in the pathogenesis of IgAN with glomerular deposition of autoantibodies recognizing overproduced, aberrantly glycosylated Gd-IgA1 [3]. KM55, a Gd-IgA1-specific monoclonal antibody generated by immunizing rats with the human Gd-IgA1 hinge region peptide, has been shown to recognize Gd-IgAl in renal biopsy tissue on formalin-fixed paraffin-embedded sections
$[4,5]$. Recent study shows KM55 is specifically detected in primary IgAN and HSPN, but not in other examined renal diseases, such as lupus nephritis, cirrhosis, or hepatitis C-related glomerular disease [6]. These findings suggest IgAN and HSPN share a common feature involving GdIgA1 in pathogenesis [6]. However, the specificity of KM55 for primary IgAN and HSPN was questioned in a more recent study demonstrating KM55 staining in incidental IgA deposition, secondary IgAN, and staphylococcal infectionassociated glomerulonephritis, albeit with weaker staining than in primary IgAN [7].

Unlike HSPN in children, clinical presentation of HSPN in adults is frequently severe [8] and associated with malignancies in up to $28 \%$ [9] of cases, including hematolymphoid neoplasm and solid tumor from primary organ of respiratory, digestive, neurologic, and urologic systems $[10,11]$. The pathophysiology of malignancy-associated HSPN remains speculative. Proposed mechanisms include abnormal production of antibodies directed against tumor 
neoantigens leading to the formation of immune complexes and similarities between tumor antigens and endothelial cell antigens. It is unknown whether Gd-IgA1 plays a role in the pathogenesis of malignancy-associated HSPN.

Here, we report detailed clinical pathological findings and immunostaining of KM55 on sequential kidney biopsies of a 35-year-old man with a clear cell renal cell carcinoma(CCRCC-) associated HSPN.

\section{Case Presentation}

2.1. Initial Clinical History, Laboratory Data, and Pathology Findings. A 35-year-old man with a past history of schizoaffective disorder controlled by medication was admitted with a bilateral, palpable, nontender rash on his legs, leg stiffness, and leg pain for 24-36 hours. The patient complained of nausea, constipation, and worsening abdominal pain. No fevers, chills, diarrhea, difficulty in urinating, shortness of breath, or cough were noted. There was no personal or family history of rheumatologic disease or hypertension. There was no history of recent ill contacts, travel, wilderness exposure, or tick bites. The pertinent laboratory results were as follows: serum creatinine, $0.75 \mathrm{mg} / \mathrm{dL}$ (Table 1 ) and serum albumin, $3.2 \mathrm{~g} / \mathrm{dL}$. Urinalysis (UA) revealed a protein albumin urine of $100 \mathrm{mg} / \mathrm{dL}$ and $7 \mathrm{RBCs} / \mathrm{hpf}$. Serological testing was negative for antinuclear antibodies (ANAs), double-stranded DNA (dsDNA), and antineutrophil cytoplasmic antibodies (ANCA). Complement levels and rheumatoid factor were normal. Renal ultrasound showed a $6.0 \mathrm{~cm}$ complex lesion in the lower pole of the right kidney.

Two days after admission, a skin biopsy of the lower shin was performed, showing leukocytoclastic vasculitis (Figure 1) with intravascular deposition of IgA and C3 (not shown), consistent with HSP. The patient was started with oral prednisone at a dose of $60 \mathrm{mg}$ daily with improvement of the rash, leg pain, and abdominal pain. In the next week, the patient's symptoms worsened with oliguria, rapid increase in serum creatinine (from 0.75 to $5.14 \mathrm{mg} / \mathrm{dL}$ ) (Table 1), and nephrotic-range proteinuria $(8.9 \mathrm{~g} / \mathrm{g} \mathrm{Cr})$. UA showed 13 $\mathrm{RBCs} / \mathrm{hpf}$ while on pulse methylprednisolone $(500 \mathrm{mg})$ intravenously (IV) for 3 days (Table 1). Dialysis was initiated, and mycophenolate mofetil (MMF) (1 g twice daily by mouth) was added. A kidney biopsy showed diffuse global endocapillary and focal segmental mesangial proliferative glomerulonephritis with IgA-dominant mesangial and capillary wall deposition, consistent with active HSPN (Figure 2). With the consideration of paraneoplastic syndrome, an urgent partial nephrectomy was performed. A clear cell renal cell carcinoma (CCRCC) pT1b was diagnosed. A few crescentic lesions were identified in the nonneoplastic kidney of the partial nephrectomy specimen (Figure 3). Renal function improved, and dialysis was discontinued seven days following the nephrectomy. The patient was discharged on the postoperative day eight on a regimen of mycophenolate and prednisone.

\subsection{Follow-Up and Sequential Kidney Biopsy Findings.} Two weeks later, the patient was admitted for shortness of breath with an increase in creatinine (from $2.58 \mathrm{mg} / \mathrm{dL}$ to
$4.76 \mathrm{mg} / \mathrm{dL}$ ) (Table 1) and oliguria caused by a leak in his nephrectomy wound. A second renal biopsy was performed at three weeks after surgical removal of the kidney tumor. The biopsy revealed diffuse cellular and fibrocellular crescents and minimal IgA deposition along glomerular capillary wall (Table 1; Figures 4(a) and 4(b)). Therapeutic plasma exchange (PLEX) was performed every other day for a total of 2 weeks, along with IV cyclophosphamide at $15 \mathrm{mg} / \mathrm{kg} \mathrm{x1}$ and continuation of the prednisone and MMF. Several days after discharge, the patient had pancytopenia, hematochezia, and epistaxis. MMF was discontinued due to concern of side effect, while prednisone and cyclophosphamide were continued with PLEX. Over the next two weeks, there was no obvious improvement in renal function. Fluid retention was controlled by renal replacement therapy. The steroid was tapered, and cytoxan was given orally instead of intravenously. During the next 4 months, clinical manifestation and laboratory tests of the patient remained stable (Table 1).

To further clarify the kidney disease etiology, determine the level of chronic kidney damage, and assess disease activity while continuing to treat with cytotoxic therapies, a third renal biopsy was performed at five months after surgical resection of the kidney tumor. The renal biopsy showed only chronic changes with fibrous crescents and negligible IgA deposition in nonsclerotic glomeruli (Figures 4(c)-4(f)). Therefore, cytoxan was discontinued, and prednisone was tapered off over the next 21 days, with continued renal replacement therapy.

\subsection{Gd-IgA1 Immunohistochemical Staining on Kidney} Biopsies. Gd-IgA1 (anti-human Gd-IgA1 (KM55) rat IgG monoclonal antibody, Immuno-Biological Laboratories Co., Ltd, at a concentration of $50 \mu \mathrm{g} / \mathrm{mL}$ ) was stained for all three kidney biopsies, one prior to partial nephrectomy and two after surgical removal of CCRCC. KM55 displayed similar staining pattern and intensity to IgA in all biopsies (Figure 5). Diffuse global granular capillary wall staining and segmental granular mesangial staining for Gd-IgA1, similar to IgA, were observed on the first biopsy, while dramatically decreased Gd-IgA1 and IgA staining along the capillary wall and in mesangium were seen on the follow-up kidney biopsies (Figure 5).

\section{Discussion}

Malignancy-associated HSPN usually affects patients older than 40 years of age (range from 29 to 79), with a male predominance [9]. The majority of the neoplasms (80\%) are detected at the time of or within 1 year after the occurrence of HSP, and $67 \%$ of the malignancies are solid tumors [9]. The renal manifestations of HSPN can range from microscopic hematuria and proteinuria only to a rapidly progressive glomerulonephritis (RPGN). Of those who undergo a kidney biopsy, 95\% show IgA deposition in the mesangial area [12-22]. The most frequent lesion in renal biopsies is proliferative endocapillary glomerular nephritis [11]. The outcome of HSPN in adults is relatively poor, and the most frequent cause of death was neoplasia (up to $27 \%$ of death) 
TABLE 1: Laboratory results before each kidney biopsy and after tumor resection.

\begin{tabular}{|c|c|c|c|c|c|}
\hline Variables & $\begin{array}{c}\text { Baseline } \\
\quad \text { (at } \\
\text { presentation) }\end{array}$ & $\begin{array}{l}\text { Before the } \\
1^{\text {st }} \\
\text { kidney } \\
\text { biopsy }\end{array}$ & $\begin{array}{l}\text { Three weeks after tumor resection } \\
\text { (before the } 2^{\text {nd }} \text { kidney biopsy) }\end{array}$ & $\begin{array}{l}\text { Five months after tumor } \\
\text { resection (before the } 3^{\text {rd }} \\
\text { kidney biopsy) }\end{array}$ & $\begin{array}{l}\text { Reference } \\
\text { range }\end{array}$ \\
\hline Sodium $(\mathrm{mmol} / \mathrm{L})$ & 140 & 134 & 144 & 138 & $133-144$ \\
\hline Potassium (mmol/L) & 4.5 & 4.0 & 3.7 & 4.6 & $3.4-5.3$ \\
\hline Chloride $(\mathrm{mmol} / \mathrm{L})$ & 105 & 101 & 115 & 104 & $94-109$ \\
\hline $\begin{array}{l}\text { Carbon dioxide (mmol/ } \\
\mathrm{L} \text { ) }\end{array}$ & 24 & 20 & 15 & 24 & $20-32$ \\
\hline Calcium (mg/dL) & 9.4 & 7.5 & 7.1 & 8.2 & $8.5-10.1$ \\
\hline Phosphorus (mg/dL) & 3.8 & 5.6 & 7.4 & 3.9 & $2.4-4.5$ \\
\hline $\mathrm{BUN}(\mathrm{mg} / \mathrm{dL})$ & 12 & 62 & 58 & 32 & $7-30$ \\
\hline $\mathrm{Cr}(\mathrm{mg} / \mathrm{dL})$ & 0.75 & 5.14 & 4.76 & 4.89 & $0.66-1.25$ \\
\hline Protein, total $(\mathrm{g} / \mathrm{dL})$ & 7.2 & 5.2 & 3.8 & 4.7 & $6.8-8.8$ \\
\hline Albumin $(\mathrm{g} / \mathrm{dL})$ & 3.6 & 1.6 & 1.0 & 1.4 & $3.4-5.0$ \\
\hline $\begin{array}{l}\text { Protein, total urine (g/gr } \\
\text { Cr.) }\end{array}$ & - & 8.91 & 9.19 & 16.99 & $0-0.2$ \\
\hline Glucose $(\mathrm{mg} / \mathrm{dL})$ & 98 & 84 & 80 & 101 & $70-99$ \\
\hline WBC $\left(10^{9} / \mathrm{L}\right)$ & 10.9 & 18.7 & 5.8 & 2.5 & $4.0-11.0$ \\
\hline Hemoglobin (g/dL) & 14.6 & 10.3 & 7.7 & 8.7 & $13.3-17.7$ \\
\hline Platelets $\left(10^{9} / \mathrm{L}\right)$ & 317 & 204 & 335 & 123 & $150-450$ \\
\hline ANA & - & $<1.0$ & - & - & $<1.0$ \\
\hline dsDNA (IU/mL) & - & 2 & - & - & $<10$ \\
\hline ANCA & - & $<1: 20$ & - & - & - \\
\hline Cryoglobulin (\%) & - & - & Neg & - & (NEG\%) \\
\hline $\mathrm{C} 3(\mathrm{mg} / \mathrm{dL})$ & - & 130 & 110 & - & $76-169$ \\
\hline $\mathrm{C} 4(\mathrm{mg} / \mathrm{dL})$ & - & 15 & 23 & - & $15-50$ \\
\hline $\operatorname{IgA}(\mathrm{mg} / \mathrm{dL})$ & - & 118 & - & - & $70-380$ \\
\hline $\operatorname{IgG}(\mathrm{mg} / \mathrm{dL})$ & - & 479 & - & - & $695-1620$ \\
\hline $\operatorname{IgM}(\mathrm{mg} / \mathrm{dL})$ & - & 25 & - & - & $60-265$ \\
\hline $\begin{array}{l}\text { Myeloperoxidase Ab, } \\
\text { IgG (AI) }\end{array}$ & - & 0.2 & 0.2 & - & $0.0-0.9$ \\
\hline $\begin{array}{l}\text { Proteinase } 3 \mathrm{Ab}, \mathrm{IgG} \\
\text { (AI) }\end{array}$ & - & 0.2 & 0.2 & - & $0.0-0.9$ \\
\hline
\end{tabular}

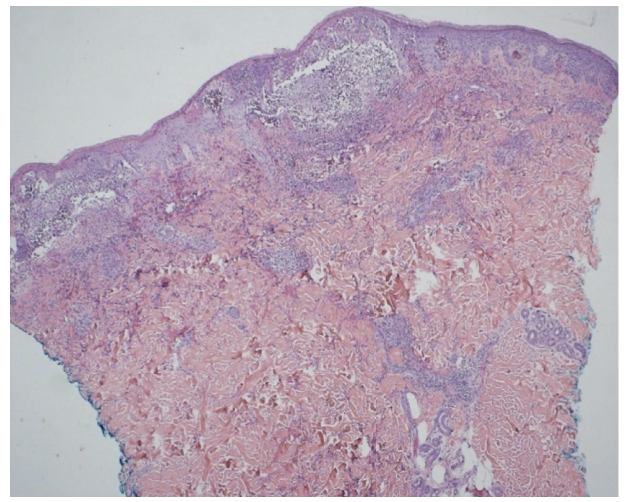

(a)

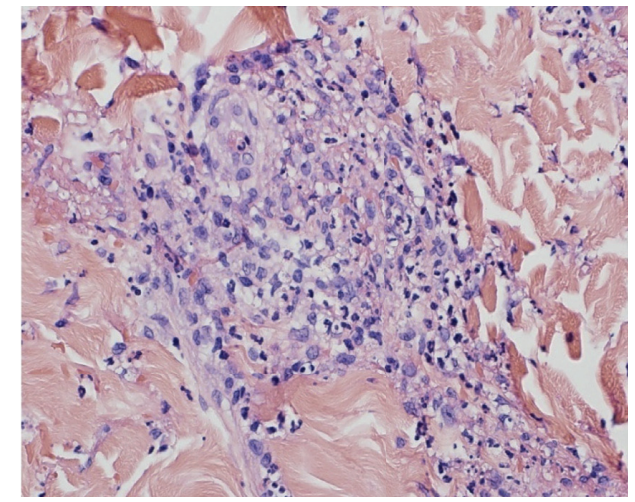

(b)

Figure 1: A punch biopsy of skin involved by a brisk acute small vessel neutrophilic vasculitis that extends from the papillary to midreticular dermis. There is prominent associated fibrinoid necrosis of vessel wall, leukocytoclasis, purpura, and epidermal degeneration. Original magnification: $4 \mathrm{X}$ in (a) and 40X in (b).

$[10-12,14,16,18,21]$ although significant improvement of renal function was reported in some cases with treatment of the cancer and corticosteroids for HSPN [13-15, 22]. The independent prognostic factors of renal outcome in patients with HSPN are proteinuria level, initial renal failure, and the histologic quantification of interstitial fibrosis and glomerular sclerosis [11].

Our patient's clinical characteristics and biopsy findings are in concordance with the reported cases in the literature. The disappearance of glomerular IgA deposits soon after 


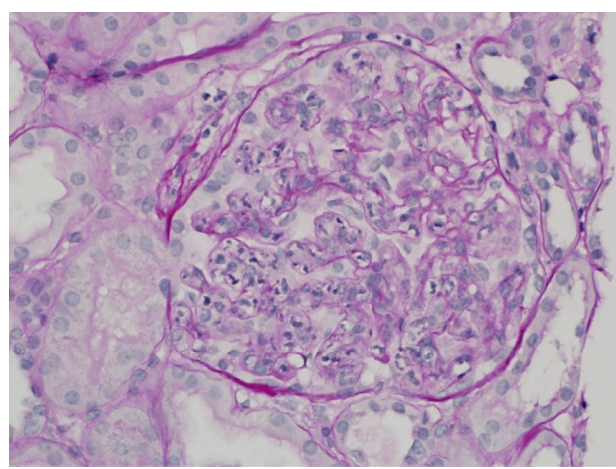

(a)

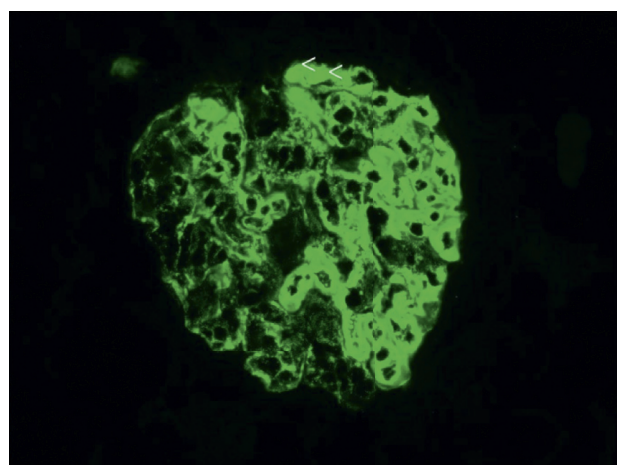

(c)

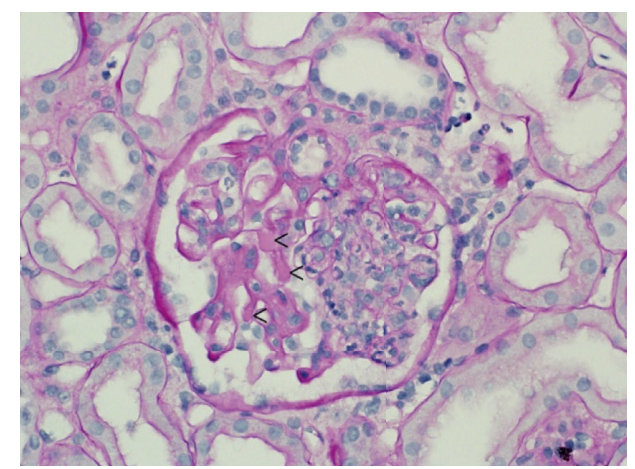

(b)

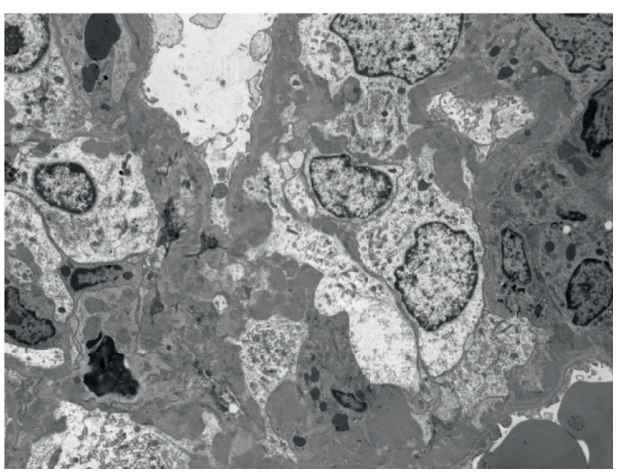

(d)

FIGURE 2: Findings of initial kidney biopsy: (a) a representative glomerulus with global endocapillary proliferation on periodic acid-Schiff stain; (b) a glomerulus with segmental mesangial and endocapillary proliferation (right side) and intracapillary pseudothrombi $(<)$; (c) immunofluorescence staining of IgA shows global granular mesangial and capillary wall staining and a few intracapillary pseudothrombi $(<)$; (d) electron microscopy shows many electron dense mesangial and subendothelial deposits with severe foot process effacement. Original magnification: $400 \mathrm{X}$ in $(\mathrm{a}-\mathrm{c})$ and $5000 \mathrm{X}$ in (d).

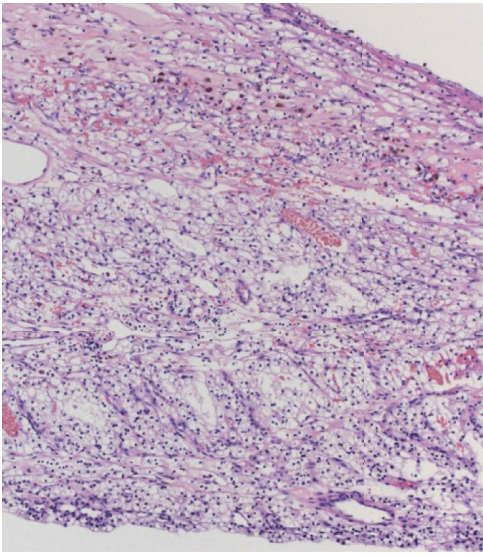

(a)

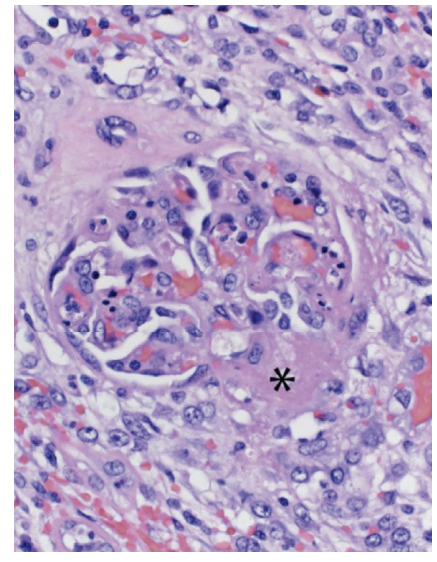

(b)

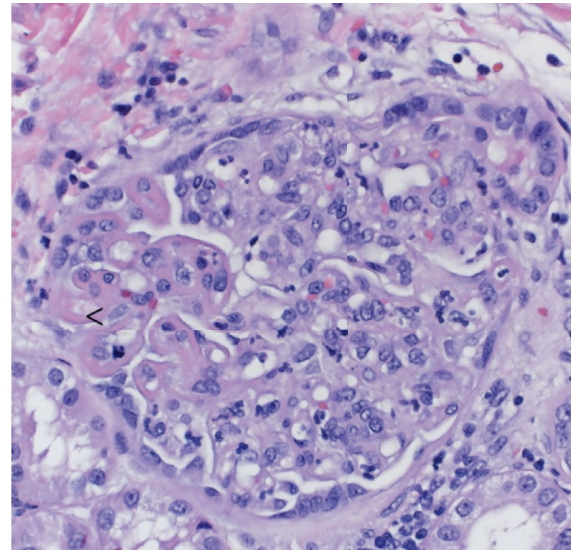

(c)

Figure 3: Clear cell renal cell carcinoma, low grade of partial nephrectomy (a). In adjacent nonneoplastic kidney, several glomeruli show segmental to global endocapillary proliferation with segmental necrotizing lesion $(*)$ in (b) and rare glomeruli show large glomerular deposits $(<)$ in (c). Original magnification: 40X in (a) and 400X in (b) and (c).

tumor removal supports that the patient's HSPN is associated with the patient's concurrent kidney cancer. The disappearance of mesangial IgA deposits on repeat biopsies has been rarely reported in children [23] and adults [24]; however, the disappearance is observed after prolonged combination therapy, two years and 77 months on average for pediatric and adult patients, respectively. Despite tumor removal and immunosuppression treatment, our patient's renal function deteriorated and the patient was on maintenance dialysis, as reported in other cases $[17,21]$. 


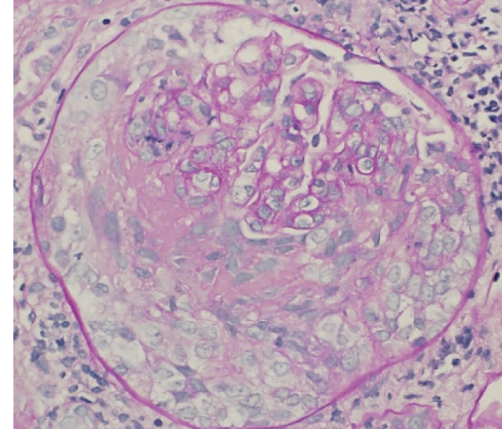

(a)

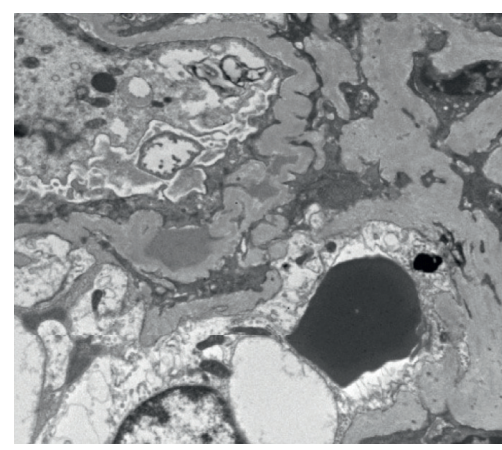

(d)

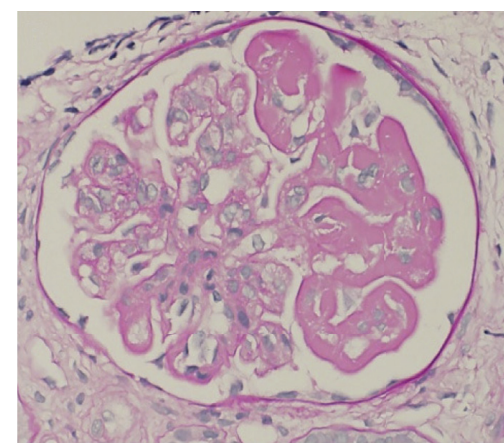

(b)

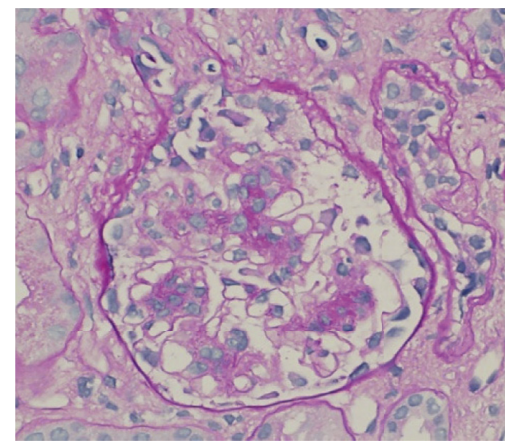

(e)

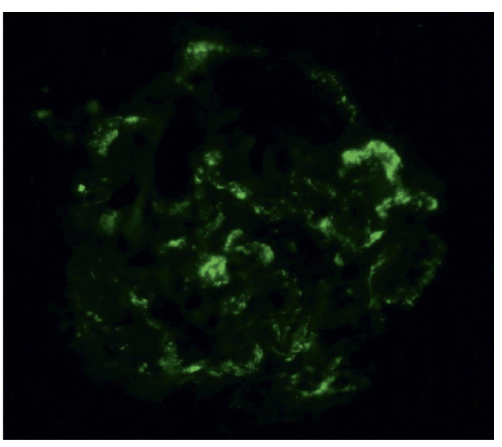

(c)

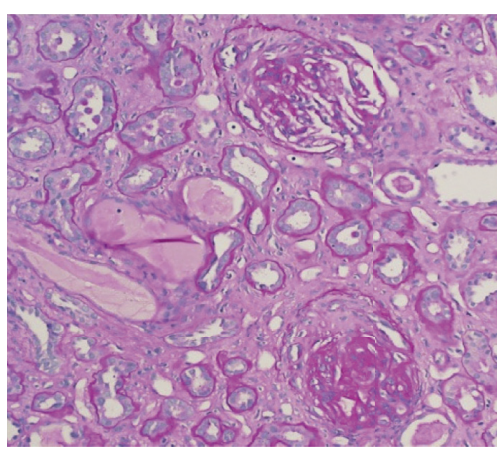

(f)

FiguRE 4: Findings of the second and third kidney biopsies, at three weeks $(\mathrm{a}, \mathrm{b})$ and five months $(\mathrm{c}-\mathrm{f})$ after surgical resection of clear cell renal cell carcinoma, respectively. Sequential biopsies demonstrate progression and transition from active lesions ( $\mathrm{a}, \mathrm{b})$ to sclerosing lesions (e, $\mathrm{f}$ ), and dramatic clearance of glomerular IgA deposits following tumor resection (c, d). (a) A representative glomerulus shows endocapillary hypercellularity and a nearly circumferential cellular crescent with necrosis. (b) Segmental large subendothelial deposits (right half of the glomerulus) are visible by light microscopy. (c) Immunofluorescence staining of IgA shows only modest glomerular mesangial and capillary wall IgA deposition. (d) Electron microscopy shows a small number of electron dense deposits mainly in mesangium. (e) Nonsclerosed glomeruli show only mild mesangial hypercellularity without endocapillary hypercellularity. (f) Representative glomeruli with fibrous crescents, one globally sclerotic (the lower glomerulus) and the other segmentally scarred (the upper glomerulus). The third biopsy shows significant chronic changes with approximately 35\% global glomerulosclerosis and additional 35\% glomeruli with fibrous crescents and segmental sclerosis. There is severe tubulointerstitial scarring. Original magnification: 400X in (a-c) and (e), 8000X in (d), and 200X in (f).

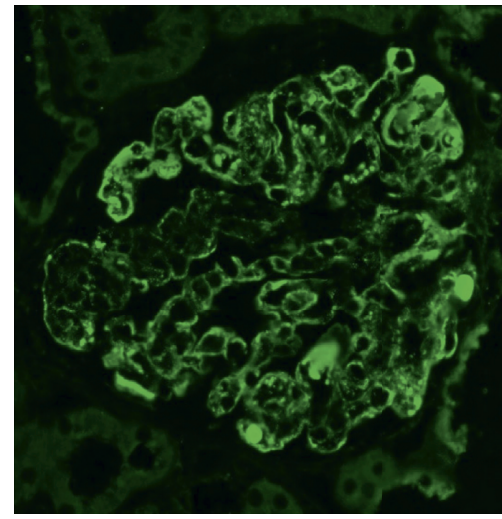

(a)

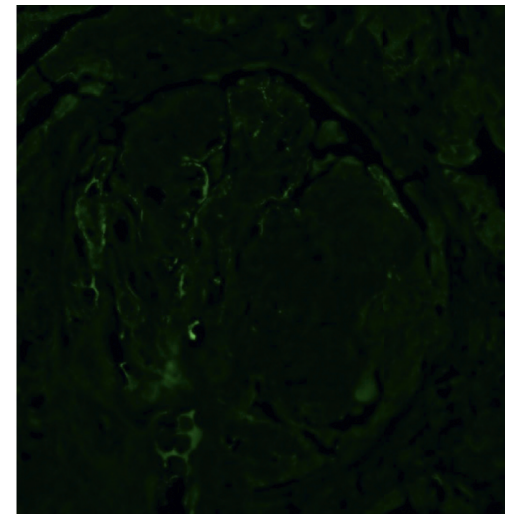

(b)

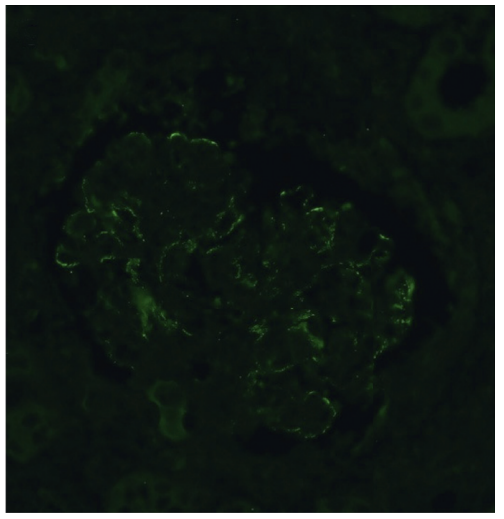

(c)

FIGURE 5: Immunofluorescence staining of KM55 shows global, granular, mesangial, and capillary wall as well as intracapillary pseudothrombi staining on initial kidney biopsy (a) and diminished/minimal glomerular capillary wall staining on sequential kidney biopsies three weeks (b) and five months (c) after surgical resection of clear cell renal cell carcinoma. Original magnification: $400 \mathrm{X}$ in all.

Gd-IgA1 plays an important role in the pathogenesis of IgAN. Gd-IgA1 is reported to increase in blood circulation and/or be deposited in renal mesangium in patients with primary IgAN and/or HSPN [25-28]. The data are sparse and controversial on whether immunostaining of KM-55, a Gd-IgA1-specific monoclonal antibody, is specific for 
patients with primary IgAN and HSPN, but not secondary form $[6,7,29]$. Here, we demonstrate glomerular KM55 staining with a similar pattern and intensity to IgA in this malignancy-associated HSPN, a secondary form of IgA deposition. These data show that KM55 staining may not be helpful in distinguishing primary from secondary HSPN. Furthermore, the KM55 staining in the sequential kidney biopsies suggests the production of autoantibodies against Gd-IgA1 molecules might be one of the pathophysiologies of malignancy-associated HSPN.

\section{Conclusions}

Here, we report KM55 staining on three sequential kidney biopsies from a 35 -year-old man with HSPN and a concurrent kidney carcinoma. Positive glomerular staining of $\mathrm{KM}-55$, an antibody that identifies Gd-IgA1, in this malignancy-associated HSPN was seen with a similar staining pattern and intensity to IgA on the initial kidney biopsy. Disappearance of glomerular mesangial IgA and Gd-IgA1 deposits was observed on two sequential kidney biopsies at three weeks and five months after surgical resection of the kidney tumor. The findings suggest Gd-IgA1, not specific to primary IgAN and HSPN, may play a role in the pathogenesis of malignancy-associated HSPN.

\section{Conflicts of Interest}

The authors declare that there are no conflicts of interest.

\section{Acknowledgments}

The authors would like to thank Dr. Patrick H. Nachman for reviewing the manuscript.

\section{References}

[1] M. H. Heineke, A. V. Ballering, A. Jamin, S. Ben Mkaddem, R. C. Monteiro, and M. Van Egmond, "New insights in the pathogenesis of immunoglobulin A vasculitis (HenochSchönlein purpura)," Autoimmunity Reviews, vol. 16, no. 12, pp. 1246-1253, 2017.

[2] M. E. Conley, M. D. Cooper, and A. F. Michael, "Selective deposition of immunoglobulin A1 in immunoglobulin A nephropathy, anaphylactoid purpura nephritis, and systemic lupus erythematosus," Journal of Clinical Investigation, vol. 66, no. 6, pp. 1432-1436, 1980.

[3] H. Suzuki, K. Kiryluk, J. Novak et al., "The pathophysiology of IgA nephropathy," Journal of the American Society of $\mathrm{Ne}$ phrology, vol. 22, no. 10, pp. 1795-1803, 2011.

[4] R. Coppo, "A new monoclonal antibody for detecting degalactosylated IgA1 as serum biomarker of IgA nephropathy," Nephrology Dialysis Transplantation, vol. 30, no. 8, pp. 1234-1236, 2015.

[5] J. Yasutake, Y. Suzuki, H. Suzuki et al., "Novel lectin-independent approach to detect galactose-deficient IgA1 in IgA nephropathy," Nephrology Dialysis Transplantation, vol. 30, no. 8, pp. 1315-1321, 2015.

[6] H. Suzuki, J. Yasutake, Y. Makita et al., "IgA nephropathy and IgA vasculitis with nephritis have a shared feature involving galactose-deficient IgA1-oriented pathogenesis," Kidney International, vol. 93, no. 3, pp. 700-705, 2018.
[7] C. A. Cassol, C. Bott, G. M. Nadasdy et al., "Immunostaining for galactose-deficient immunoglobulin $\mathrm{A}$ is not specific for primary immunoglobulin A nephropathy," Nephrology Dialysis Transplantation, 2019.

[8] J.-C. Davin, "Henoch-Schönlein purpura nephritis: pathophysiology, treatment, and future strategy," Clinical Journal of the American Society of Nephrology, vol. 6, no. 3, pp. 679-689, 2011.

[9] E. Pertuiset, F. Lioté, E. Launay-Russ, F. Kemiche, I. CerfPayrastre, and A.-M. Chesneau, "Adult Henoch-Schönlein purpura associated with malignancy," Seminars in Arthritis and Rheumatism, vol. 29, no. 6, pp. 360-367, 2000.

[10] P. S. Kellerman, "Henoch-Schönlein purpura in adults," American Journal of Kidney Diseases, vol. 48, no. 6, pp. 1009-1016, 2006.

[11] E. Pillebout, E. Thervet, G. Hill et al., "Henoch-Schonlein purpura in adults: outcome and prognostic factors," Journal of the American Society of Nephrology, vol. 13, no. 5, pp. 12711278, 2002.

[12] R. K. Kim, A. R. Thomas, M. E. Card, D. Malhotra, and J. L. Holleck, "Four cancers and a rash: Henoch-Schönlein purpura," The American Journal of Medicine, vol. 130, no. 10, pp. 1158-1160, 2017.

[13] I. Mimura, A. Tojo, S. Kinugasa, T. Fujita, and H. Uozaki, "Renal cell carcinoma in association with IgA nephropathy in the elderly," The American Journal of the Medical Sciences, vol. 338, no. 5, pp. 431-432, 2009.

[14] J. M. Zurada, K. M. Ward, and M. E. Grossman, "HenochSchönlein purpura associated with malignancy in adults," Journal of the American Academy of Dermatology, vol. 55, no. 5, pp. S65-S70, 2006.

[15] N. Akizue, E. Suzuki, M. Yokoyama et al., "Henoch-Schönlein purpura complicated by Hepatocellular carcinoma," Internal Medicine, vol. 56, no. 22, pp. 3041-3045, 2017.

[16] J. Podjasek, D. Wetter, M. Pittelkow, and D. Wada, "HenochSchönlein purpura associated with solid-organ malignancies: three case reports and a literature review," Acta Dermato Venereologica, vol. 92, no. 4, pp. 388-392, 2012.

[17] P. Blanco, R. Denisi, P. Rispal et al., "Henoch-Schonlein purpura associated with segmental and focal proliferative glomerulonephritis in a patient with Hodgkin's disease," Nephrology Dialysis Transplantation, vol. 14, no. 1, pp. 179-180, 1999.

[18] P. Arrizabalaga, A. Saurina, M. Solé, and J. Bladé, "HenochSchönlein IgA glomerulonephritis complicating myeloma kidneys: case report," Annals of Hematology, vol. 82, no. 8, pp. 526-528, 2003.

[19] Y. H. Hong, "Renal cell carcinoma presenting as HenochSchönlein purpura with leukocytoclastic vasculitis, hematuria, proteinuria and abdominal pain," Rheumatology International, vol. 30, no. 10, pp. 1373-1376, 2010.

[20] T. Posas-Mendoza, D. Lucuab-Fegurgur, and J. Roberts, "Adult onset Henoch-Schonlein purpura associated with a metastatic malignancy of unknown primary origin," Hawai' $i$ Journal of Medicine \& Public Health, vol. 77, pp. 243-245, 2018.

[21] S. Joshi, S. Pillai, A. Chakera, and Y. C. G. Lee, "HenochSchonlein purpura in mesothelioma," Respirology Case Reports, vol. 2, no. 4, pp. 138-140, 2014.

[22] M. S. Y. Ng, L. Francis, E. Pillai et al., "Paraneoplastic immunoglobulin A nephropathy and associated focal segmental glomerulosclerosis in asymptomatic low volume B-cell lymphoma-a case report," BMC Nephrology, vol. 19, p. 224, 2018. 
[23] Y. Shima, K. Nakanishi, K. Kamei et al., "Disappearance of glomerular IgA deposits in childhood IgA nephropathy showing diffuse mesangial proliferation after 2 years of combination/prednisolone therapy," Nephrology Dialysis Transplantation, vol. 26, no. 1, pp. 163-169, 2011.

[24] O. Hotta, T. Furuta, S. Chiba, S. Tomioka, and Y. Taguma, "Regression of IgA nephropathy: a repeat biopsy study," American Journal of Kidney Diseases, vol. 39, no. 3, pp. 493-502, 2002.

[25] Z. Moldoveanu, R. J. Wyatt, J. Y. Lee et al., "Patients with IgA nephropathy have increased serum galactose-deficient IgA1 levels," Kidney International, vol. 71, no. 11, pp. 1148-1154, 2007.

[26] Y. Wada, K. Matsumoto, T. Suzuki et al., "Clinical significance of serum and mesangial galactose-deficient $\operatorname{IgA} 1$ in patients with IgA nephropathy," PLoS One, vol. 13, Article ID e0206865, 2018.

[27] W. J. Placzek, H. Yanagawa, Y. Makita et al., "Serum galactose-deficient-IgA1 and IgG autoantibodies correlate in patients with IgA nephropathy," PLoS One, vol. 13, Article ID e0190967, 2018.

[28] M. Mizerska-Wasiak, Ł. Gajewski, K. Cichoń-Kawa et al., "Serum GDIgA1 levels in children with IgA nephropathy and Henoch-Schönlein nephritis," Central European Journal of Immunology, vol. 43, no. 2, pp. 162-167, 2018.

[29] M. Wang, J. Lv, X. Zhang, P. Chen, M. Zhao, and H. Zhang, "Secondary IgA nephropathy shares the same immune features with primary IgA nephropathy," Kidney International Reports, vol. 5, no. 2, pp. 165-172, 2020. 